\title{
Operations Research in Public Health
}

\author{
STIG ANDERSEN
}

$\mathrm{T}$ HE SPECTACULAR progress of public health in certain parts of the world during the last century, and notably in the last half century, was a result of a cumulative interaction between a large number of factors, many of them associated with economic progress and the development of science. The prospering society could afford to buy more and more public health services, and improved public health in turn accelerated economic progress. At the same time, the technical means of the public health services were created and developed more or less in step with the increasing capacity of a prosperous society to apply them.

The situation in the less industrial countries by mid-twentieth century corresponds in some respects to the situation a hundred years earlier in Europe and the United States. Their economies cannot afford to buy adequate public health service, and the state of their national health hampers progress. The decisive difference, however, is that a large part of the inventions and experience in techniques gained elsewhere are available now to all nations to apply in logical systems as and when possible.

This relative preponderance of technical knowledge over economic capacity is the social fact which necessitates a new type of research. The research which is needed most at this time is not inventive. These societies are not in pressing need of new techniques and new in-

Dr. Andersen prepared this paper, which was published in the October 1963 issue of the Indian Journal of Public Health, when he was serving as senior WHO officer at the National Tuberculosis Institute, Bangalore, India. Since October 1963 he has been deputy resident representative of the United Nations Technical Assistance Board and deputy director of the UN Special Fund in the Philippines, with offices in Manila. ventions. Their demand is for systems composed of currently available techniques which give the optimal utilization of scarce economic resources. Research that satisfies this demand can be called operations research, at some risk of criticism from other employers of the term.

After a start in the military field in the 1940's, the techniques of operations research have been developed mainly in the field of industrial management. There is little likelihood that operations research in public health can reach a stage, for many years to come, where it can utilize directly many of the mathematical programing techniques which have been developed for military and industrial purposes. However, the basic concept of research into total systems which, in principle, can be translated into mathematical models can and should be adapted to the needs of public health services research. The name operations research implies research into some or all aspects of conducting or operating a system, a business, or a service, while treating the system as a living organism in its proper environment; thus it distinguishes itself from laboratory research.

Following are the major phases in operations research, adapted from Churchman and associates (1) and Houlden (2) :

1. Formulating the problem, including definition of the objectives.

2. Collection of data relevant to the problem.

3. Analysis of data to produce a hypothesis and a mathematical model to represent the system under study.

4. Deriving solutions from the model.

5. Choosing the optimal solution and forecasting results.

6. Testing the optimal combination of interventions, with controls built into the system to keep continuous check on the hypotheses. 
7. Recommending implementation of the solution, including the control system.

The concept of an operations research team is an important aspect of the process. No one single person possesses all the necessary skills and experience to conduct worthwhile operations research. Usually a team in industry comprises scientists in the fields of mathematics, statistics, economics, and engineering, in addition to experts in the special field under study. Some teams also include a professional logician or a specialist in the science of scientific methods.

\section{Applications to Public Health}

The need for applying operations research to public health has been recognized by a few public health administrators in the United States and in the United Kingdom, for example, Committee on Enquiry (.3) and Bailey $(4,5)$, particularly with respect to hospital planning.

Undoubtedly many public health administrators use methods implicitly akin to operations research. But $I$ hope to demonstrate that explicit adoption of the operations research approach would benefit public health services of developing economies. It may be seen that operations research may be applied not only to the solution of detailed problems within the services but, more importantly, to larger systems within them and to the entire system of the public health service in the nation.

A modest attempt to apply operations research to one system within India's Public Health Service is being made by India's $\mathrm{Na}$ tional Tuberculosis Institute in developing the national tuberculosis program. The institute is only at the beginning of its efforts, and only a few aspects have been described in published papers. Andersen and Piot (6) summarize the operations research approach of the N'TI. Raj Narain ( 7$)$ summarizes the extent of the problem; Waaler and associates ( 8 ) attempt a first formulation of an epidemetric model, and Banerji and Andersen (9) and Andersen (10) deal with certain sociological and economic aspects. A provisional "optimal combination of interventions" is suggested by Piot (11).

The present paper attempts to foresee some of the types of problems which an operations research team in public health would face. These are divided into the seven major phases of operations research listed above, with the Indian experience as background.

\section{Formulation of the Problem}

The first and perhaps one of the most formidable challenges to the operations research team is the precise and explicit formulation of the problem. What is the system under study? Does it comprise the entire health field in the nation? Or can the team at least confine itself to that part which is under direct public control? How does the team define the boundaries of its field towards other public undertakings, the expenditure for which can be considered alternative to expenditure for public health? Can it evaluate public health output without comparisons with results of alternative investments? It is not unlikely that it may be found necessary to confine the first stages of a system analysis to one or a few local areas, and perhaps even, to begin with, to a given total budget, so as to reduce the total number of variables.

Even if, by this and other means, the system under study is brought down to manageable proportions, the team is left with an equally arduous task: the definition of objectives. At first glance it may appear puzzling that this should be part of the researcher's function. It is usually, and rightly, considered outside the province of the scientist, as a scientist, to decide or interfere with the objectives of a service, which are determined by the executive and ultimately by the people and its elected assemblies. However, in modern complex societies the popular will and even the executive's objectives are not necessarily transparent, and they are usually difficult to translate into scientific terms.

Conceivably the administrative and political heads of a public health service could be assumed to want "the maximum utilization of the given resources towards the promotion of health among the people," but the operations research team would require far more specific objectives than that. A major obligation of the team in the first stages of its work is to guide the executive toward very specific definitions of objectives. (If the team never achieved anything else, this would in fact be an extraordinarily valuable contribution.) 
The team must stimulate the executive to define the objectives by presenting the logical alternatives. For example, taking a starting point in the above statement of objective, What is meant by "given resources"? Cannot the resources planned to be expended on health services be increased or reduced if the expenditure can be shown to be more or less profitable than assumed? What is meant by "promotion of health"? One might particularly wish to emphasize one of two entirely different aspects: (1) the state of health itself in a certain distribution in the population, which again might be viewed according to $(a)$ absence of illness and $(b)$ presence of positive health, or $(\mathscr{P})$ the existence in reasonable proximity to the thus distributed population of a confidence-inspiring health service. Again, "among the people" must be far more sharply defined; assuming that equality is a guiding principle, are qualifications to the general equality principle to be considered permissible, for example, emphasis on highly productive groups or on children versus old people? Among the most difficult problems are those of the dynamics of the objectives: Should target dates be set? Or should the formulations be more dynamic and relate to the development over a period? If so, which period? And should they be related to the concomitant economic, demographic, and other social development?

Already at this stage the team will have to start considering the terms of the solutions, particularly on the output side. When the teamand the executive-have agreed on a set of general objectives, these have to be specified, perhaps in the form of indices, some of which probably can only be formulated in the course of data collecting.

\section{Collection of Data}

The collection of data is inextricably related to the formulation of objectives and the formulation of the hypothesis. Data collection, no doubt, is bound to be extensive, but it must also be strictly economical and strictly relevant to the objectives and the hypothesis. Data collection in present-day public health services research is perhaps relatively overdeveloped. A wealth of material, no end of official reports, and a considerable amount of scientific data be- come available every year, but this material is rarely, if ever, related to clearly formulated objectives and hypotheses, and it is therefore of far too little value for decision making. For this very reason, the operations research team will have to supplement existing data considerably. But it should only collect data that are strictly necessary for its other functions, and it should not be hesitant about making informed guesses when it finds that data collection on a particular subject is out of proportion in cost to the importance the data will have in the total system. Naturally, the team will rely extensively on sampling techniques. This is particularly true for a large part of the output functions, where a good deal of the information is to be collected among the general population.

On the input side, cost of services, training of personnel, handling medical stores, administrative and technical operations, the number of observations will often be too small for sampling. The major part of the data to be collected under this phase will be on the system as it now works and the results that are now obtained. But under many circumstances it will probably be necessary to supplement these data with a limited amount of data of a more experimental type. Such public health "experiments" have for example been found useful by India's National Tuberculosis Institute, which is conducting a series of comparisons of organizational approaches to tuberculosis control in a number of primary health unit blocks in southern India. The detailed protocols for these so-called operational investigations are available (12).

For its data collection functions the research team will have to employ special investigators. Depending on the fields in which data are particularly lacking, medical officers, various kinds of paramedical technicians, accountants, social investigators, and demographic investigators may be needed. Most of these specialists must be given special courses in interviewing techniques.

One phenomenon must be mentioned in this context: the effect of observation on that which is observed. This is a difficult factor to deal with in all scientific inquiry, but it is particularly disturbing in operations research where experimental conditions camnot, in fact shall not, be established. 
This observer effect must be kept constantly in view, both in the data collection and the test runs (where all devices must be employed to minimize its influence) and in the analysis, where the effect which nevertheless remains must be accounted for.

\section{Analysis and Hypothesis Formulation}

'The formulation of the hypothesis or the construction of the model to represent the system under study may be considered the pivotal procedure of operations research in the fields where it is now relatively highly developed. By far the largest part of existing literature on operations research deals with the mathematical and statistical aspects of this phase of operations research. However, Eddison and associates (13) have reviewed operations research in industrial management in largely nonmathematical terms.

There is also no doubt that the pioneers in operations research in public health must strive, within a few years, to reach the stage where at least simple models depicting a public health service can be constructed. Such models will probably be input-output models of the type which are particularly employed in econometric research. However, for a system as complex as an entire national health service, with inputs that can only with the greatest difficulty be translated into common terms, and particularly with outputs of which the units vary from, say, average nutritional status of a population to satisfaction of the average health service consumer, it is unlikely that comprehensive and useful models, mathematically formulated, will result from the first few years of the research team's efforts. This does not by any means defeat the purpose of adopting operations research techniques in public health.

First, for some time, invaluable contributions can be made by the other phases of the research: the careful formulation of the problem and the objectives, the purposeful, economical collection of data, the precisely formulated and carefully evaluated test runs.

Second, while it may not be possible initially to formulate the model or the hypotheses mathematically, the operations research team can think mathematically, that is, logically, in mak- ing the formulation under this phase. Its members will think in terms of hypotheses, assumptions, parameters, and prognoses precisely expressed and systematized. By committing themselves they will expose themselves to the test of rerification.

The model is a simplified explicit description of the existing services, the elements and factors of which it consists, and the relationships between them. It summarizes the input of resources: money, material, personnel training; preventive, curative, and educational services; and administrative, decision-making, and evaluating machinery; it outlines the geographic and functional distribution of such resources. It summarizes output partly with regard to operational achievement, public participation in services, and numbers of relevant health personnel actions (again geographically and functionally distributed) and partly in relation to fulfillment of declared objectives, including disease control, health promotion, demographic change, consumer satisfaction, and economic effects.

The construction of such a model, even if vastly simplified, is in itself complex. However, the research team cannot be satisfied before it has at least broadly outlined the relationships of the interactions of the public health system with the partly supplementary, partly competitive relationships between investments in public health and alternative investments.

The operations research team will, certainly in the first phases of its work, severely limit the scope of the model. As mentioned under definition of objectives, the team would perhaps also start with the assumption that present allocation of resources to the total public health service is an unchangeable parameter. Furthermore, it would restrict the measurement of objectives fulfillment in the model to a few simple indices. Finally, but not least, the team would attempt to construct the whole system on the basis of a manageable number of key variables. One of the several functions of the team is the selection of what is to be considered, at least provisionally, the key variables of the system. In such a complex system as a health service, no model or hypothesis can ever by expected to comprise all the innumerable interdependent variables of which the system consists. Among 
them, the team must choose the key variables, that is, the variables which if changed bring about the largest effect in the total system.

Though observation plays a considerable role when this choice is made, there can be no doubt that insight and more or less intuitive under. standing of the system will be of decisive importance. This is the situation where all team members, with wisdom and patience, even humility, must sort out what is true insight from what is mere prejudice.

It is in the formulation of these hypotheses that teamwork is put to its acid test. There can be no strict delimitations of the functions of each team member. The logistics and methodology may well be largely contributed by the mathematician and the statistician, and the material knowledge by the public health administrator, the epidemiologist, the economist, and the sociologist, but unless they all gradually get a considerable grasp of each others' subjects they will be unable to make their full contribution.

Examples of factors which would be part of the model are:

Input: The total health budget, its geographic and functional breakdown, inflow and outflow of personnel, quantity and quality of training, inflow, storage, and consumption of durable and nondurable goods, and distribution of all types of health services and institutions.

Output: Operational achievements seen from the side of the services, including participation and attendance, vaccinations performed, number of childbirths assisted, wells constructed, drugs distributed. Otherwise the output part of the model can probably afford to be rather sketchy and give four or five indices, for example, demographic: crude or age-specific death rates and birth rates; epidemiologic: prevalence of two or three major diseases; and educational: at least one index of health educational status.

\section{Derivation of Solutions From the Model}

The manipulation of the model consists of a long continuous series of theoretical input changes in the model and calculation of the probable output changes. For example, input changes range from replacement of female health visitors with males and simplification of procedure for indenting supplies to major shift of emphasis from curative to preventive services and radical reforms in the organizational machinery or in the training of health personnel. The solutions theoretically carried out-on paper and in digital computers-will have the form of combinations of interventions in the existing system. The number of possible combinations of interventions, naturally, is virtually infinite.

However, several factors will restrict the number of combinations deemed worthy of study. First, the team's choice of key variables in the system (which may, admittedly, be revised in the course of the process of deriving solutions) will limit the number of even theoretically possible solutions. Second, the economist will constantly keep the team in check as to what is economically feasible; the public health administrator will have a similar function on administrative subjects; the epidemiologist will restrain enthusiasm, for example, concerning the possibilities of eradication of specific diseases; the sociologist will no doubt often have to remind the team of what is socially, psychologically, and politically acceptable; and the statistician and the mathematician will, among other tasks, certainly see that the imagination of the team does not transgress reasonable limits of calculability.

Finally, and most importantly, the common sense of all team members will restrict the field of theoretical study to sufficiently few combinations to make it possible to derive solutions before the administrators become too impatient about the results.

When this is said, however, one should not forget that it is exactly in the play of the creative imagination of the team, together with its scientific restraint, that hope lies for considerable improvement of the system under study. Within reasonable limits of realism and practicability, the team should play a free game with the multiple factors and relationships involved, combining and recombining until theoretically optimal combinations are approached. This "game" will consist of an iterative process of changing the value of a small number of variables while keeping the remaining variables constant. In this process, the team will pursue 
a number of intervention combinations, appearing virtually equally good, until it ends up with perhaps 5 or 10 combinations of which the output results seem to be of comparable magnitude.

\section{Choice of Solution and Prognosis}

Thus arriving at a limited number of intervention combinations, the team faces the choice of one of these solutions for testing. Under some circumstances the team may find it desirable and possible to test more than one solution.

Before making this choice the team consults the executive. The various intervention combinations are likely to differ from each other, particularly in two major respects: $(a)$ the degree of departure from the existing system will vary, and $(b)$ the solutions will differ in the degree to which they are calculated to fulfill each of the three, four, or five major objectives.

The team is now in a position to present the executive with a set of specified proposals for interventions, with details of changes needed in the system and consequences of these changes.

Once the choice of one or several combinations of interventions is made, the team works out the details of implementation of input changes and details of the prognosis of results, operationally as well as in terms of fulfillment of objectives.

These details should in the first instance be worked out only for the test run; they should specify the exact timetable of implementation. One would hazard to guess that much of the intervention would emphasize changes in training and would comprise a series of manuals detailing the functions of all personnel engaged in the public health services in the area.

Crucial to the solution is the prognosis. This prognosis will partly forecast the change in operational achievements and partly, the change in results (mostly in indices) with respect to the declared objectives. The task is, at least in principle, relatively simple as far as operational achievements are concerned: for example, specified changes in attendance at outpatient departments, a certain change in turnover of such and such drugs in the medical stores depot, a given number of new closed and chlorinated wells in so many villages dis- tributed according to a given plan over the area.

But more difficult by far, both principally and practically, is the team's task of forecasting the results with respect to the general objectives, as it camnot afford to omit any of the major aspects of health and disease. For example, while incidence of smallpox may not necessarily figure in the model describing the existing situation, the forecast cannot exclude an opinion on the prospect of smallpox outbreaks.

One of the obstacles for the output forecast is the relatively slow development of health status, health consciousness, health action, or health habits. Even rather radical changes in several aspects are not observable in less than 5 or 10 years. The team will therefore probably have to select a range of indices with varying reliability and varying sensitivity so that changes in at least some of them are observable after a rather short time, say 2 years. If these indices are then, as one would expect, relatively unreliable, one must await their gradual confirmation by more reliable, but less sensitive, indices.

The form of the prognosis will largely be statistical and conditional, with results expressed, for example, as 19 in 20 chances of a value being within certain limits provided the operational achievement attains a given value and within certain other limits if the operational achievement attains such other value.

\section{The Test Run}

The test run is not a central part of operations research in industrial management, but it probably should play a key role in the application of operations research to public health services. Tnlike industrial researchers, the team will be confronted with a large number of factors which will not be within their ambit of control, although the demand for health services is probably as foreseeable as the demand for, say, nylon stockings.

The most important characteristic of the test run is that, while limited geographically, it is true to life in every possible aspect. Of course, the observer effect will play a role; it is also inevitable that the personnel executing the test will know that they participate in a special program and that this will influence their behavoir. 
These factors must be rigorously controlled, and in the evaluation of the test run they must, as far as possible, be taken into consideration.

Obviously, the test run is in no way spectacular, no showpiece. The test operators are, with as little fuss as possible, given new instructions to follow. In many cases these may not be strikingly different from existing ones, though probably often more explicit. The test run will also normally be within, or not significantly exceed, previous budgets, since the operations research team will always keep strictly within the limits of what is nationally applicable. Indeed, it is conceivable that the team will have arrived at a lower budget, put to better use than the previous one.

The test run area should be of manageable size, but it should be sufficiently large to provide observations for proper statistical inference.

The major difference between the test run and the final national implementation is that the former has a more intensive control system. Even the final implementation will have, of course, a carefully planned control system, but to derive full benefit from the test run the team will require a particularly good apparatus through which information on all aspects of the operations and their results are fed back. This feedback will continuously be analyzed and compared with the prognosis. Whenever key variables attain values outside the predetermined limits of variation, these new values must be fed into the model and the hypotheses, and the model solved anew. The question will soon arise as to what extent to allow changes in the test run programs, if they are wide of the mark. In principle, the test run should be allowed to run itself out without modification and new test runs in new areas applied if need be, but the team may have to compromise.

Although the operators and operations should themselves report on their performance, the test run will need additional staff to conduct sample investigations of the results (mainly interviews of population) and even local statistical assistance to cope with the data acquired.

\section{Recommending Implementation}

The last link in the chain of operations research is application of the solution to the whole system and establishment of evaluation machin- ery with an apparatus for new decision making when the key variables change beyond predetermined limits. In industry (and in the military field), such implementation follows more or less automatically after the final solution has been derived and tested, though even here the stockholders may insist on having their last say. In a public service, there may well be other obstacles to a rational solution.

This rationality may be questioned by the executive or by the public. Even when the rationality is recognized, the solution may be considered politically unacceptable to special groups or certain political parties, including the party in power. However, in principle, such factors should already have been taken into consideration by the research team. This is one of several justifications for the inclusion of a sociologist. Failure to have the scheme accepted can rightly be blamed on a team that did not appreciate the political climate in which it worked. For example, it is of little use if the team concludes that the whole health service, including general practitioners, should be nationalized, if the feelings and power of the medical profession or the public are such that this part of their solution is not feasible.

The recommendation for national implementation would probably in many cases, notwithstanding a successful test run, be in the form of recommendation for gradual extension, with a large pilot area to begin with, where the realism of the test-run-modified solution is put to a final test.

\section{Minor Uses of Operations Research}

Operations research is holistic. However, whole systems usually consist of many smaller ones. A national public health service is itself only a sector of a whole socioeconomic system, and the public health service in its turn comprises numerous large and small sectors and units, within many of which one can well visualize good use being made of operations research. Work on subsystems has, it would seem, far lower priority than the efforts described in the preceding paragraphs, but a team working in a country on a major effort might find it worthwhile to gain experience from small systems. 
One example of a subsystem which would no doubt be fertile ground for operations research in many countries is the medical stores system. Considerable loss in operational efficiency is sustained in some countries because the medical stores take months, even years, to expedite indents, and this often occurs at the same time that the stores have to dispose of, and occasionally even destroy, items which have become obsolete.

The team might also try to solve subordinate problems within the main system before or as part of the whole solution. Examples are optimal size of area and population to be covered by midwife, smallpox vaccinator, or basic health unit; ideal vehicle for local health workers; administrative management solutions leaving maximum time for technical personnel to use their skills; architectural design of hospitals and health centers; queueing in outpatient departments and hospital waiting lists; integration of specialized services developed on an emergency basis outside the general health services.

\section{The Operations Research Team}

Operations research can be a more or less continuous process, or it can be a valuable onetime effort. Once a team has gone through the procedure described above, the service will presumably have been considerably improved. For a small national health service, such a procedure, or perhaps gradually a more simplified one, could be repeated at economical intervals. For a large country, for example the three or four largest in Asia, operations research could be perpetual. The team here would be employed, perhaps equally, in overall systems research and in work on subsystems.

With such a variation in the requirements, it is difficult to suggest general rules for the size and composition of the operations research team. However, some features would be more or less common to all such teams. The minimum composition is probably a public health administrator, an epidemiologist, a mathematician, a statistician, and a social scientist. Larger teams should probably also comprise a sanitary engineer and an educationist, and the general social scientist should be replaced by an economist and a sociologist. To these must be added, because the team must have its own data collection apparatus and test-run evaluation system, a number of investigators of various categories, perhaps 10, 20, or more, and perhaps as many junior statisticians and statistical clerks.

Finally, if the team cannot rely on outside assistance in computer programing, it must have its own programer, with full-time use of statistical processing machinery and part-time use of a digital computer.

Many skeptics will say, "This is just common sensc, with a superstructure of fine new words." Operations research is very little but common sense. This is perhaps even more true when it is admitted that in the first stages of applying operations research in public health services, mathematical models may be of limited relevance. The essence of operations research is that logical thought, combined with careful observation and methodological analysis, should form the basis for decision making. Adoption of such a principle may not appear revolutionary, but in an irrational world the public health service that carries it out to its full logical consequence, abiding by its sometimes exacting commands, would be likely to find itself vastly improved. The methodological study of alternative courses of action can, of course, be given many names; probably most operations researchers would be proud to have their science known as the science of common sense.

Other skeptics assert that operations research can never replace experience. Human experience is a more or less unconscious collection of data and analysis of data by an individual or a group of individuals. But experience is often unreliable, is often narrow and biased. Scientific methods can remove the bias and insure that data are properly systematized to give a true picture of the past and a more reliable forecast of the future, including the probable outcome of intended executive action. They can also give a measure of the so-called imponderables by calculating probabilities of occurrences.

\section{REFERENCES}

(1) Churchman, C. W., Ackoff, R. L., and Arnoff, E. L.: Introduction to operations research. John Wiley \& Sons, Inc., New York, 1957. 
(2) Houlden, B. T., editor: Some techniques of operational research. Hazell Watson \& Viney Ltd., London, 1962.

(\$) Committee of Enquiry (Guillebaud Committee) into the Cost of the National Health Service: Report. Her Majesty's Stationery Office, London, 1956.

(4) Bailey, N. T. J. : Operational research in hospital planning and design. Operat Res Quart 8: 149, 1957.

(5) Bailey, N. T. J.: Operational research. In Society, problems and methods of study, edited by A. T. Welford, M. Argyle, D. V. Glass, and J. N. Morris. Routledge \& Kegan Paul Ltd., London, 1962.

(6) Andersen, S., and Piot, M.: The operations research approach. In Souvenir of the 18th AllIndia Tuberculosis and Chest Diseases Workers' Conference, Bangalore, 1962. (y) Raj Narain: Size and extent of the tuberculosis problem in urban and rural India. Indian $\mathbf{J}$ Tuberc 9: 147, June 1962.

(8) Waaler, H. T., Geser, A., and Andersen, S.: The use of mathematical models in the study of the epidemiology of tuberculosis. Amer J Public Health 42: 1002, June 1962.

(9) Banerji, D., and Andersen, S.: Bull WHO. In press.

(10) Andersen, S.: Some aspects of the economics of tuberculosis in India. Indian $\mathrm{J}$ Tuberc $9: \mathbf{1 7 6}$, June 1962.

(11) Piot, M.: Outline of a district tuberculosis programme. Indian J Tuberc 9: 151, June 1962.

(12) National Tuberculosis Institute: Protocols for operational investigations. Bangalore, India, 1961. Mimeographed.

(13) Eddison, R. T., Pennycuick, K., and Rivett, B. H. P.: Operational research in management. English Universities Press, Ltd., London, 1962.

\section{No Truce for Tuberculosis}

The 1964 theme for World Health Day, observed April 7, is "No Truce for Tuberculosis." Infectious tuberculosis afflicts at least 15 million persons in the world and still claims more than 3 million lives a year, according to Dr. M. G. Candau, Director-General of the World Health Organization.

While tuberculosis mortality has declined in the economically developed countries, the decline in morbidity has been less marked. In no country in the world has the number of children infected with tuberculosis before they reach the age of 14 years fallen below 1 percent, Dr. Candau indicated. This is the criterion set up by the WHO Expert Committee on Tuberculosis as a basis for determining whether a nation has eliminated tuberculosis as a public health problem. In the less developed areas, 70 percent of the children may be infected before they reach the age of 14 . Probably between 2 and 3 million new cases occur in the world each year.

In his statement supporting the campaign, Dr. Luther L. Terry, Surgeon General of the Public Health Service, observed that in view of the global toll, no nation can remain unconcerned about the social consequences of this costly disease. "Today, more than ever, we have not only the theoretical but the practical capability of making a real impact on tuberculosis." 


\section{Campaign on Teenage Venereal Disease}

The rate for early infectious syphilis in teenagers in Los Angeles is $21 / 2$ times greater than for this group in the United States as a whole. In Los Angeles teenagers, early infectious syphilis has increased more than 725 percent during the past 7 years. There are more than 230,000 teenagers in the Los Angeles City school system, 48 percent more than 7 years ago.

Therefore, aided by a recent grant from the Public Health Service, the Los Angeles County and City Health Departments have focused a newly initiated interlocking disease control program on providing venereal disease information to teenage pupils. The grant of approximately $\$ 35,000$ for the venereal disease control project provides funds for one professional person for the county and one for the city, plus salaries for clerical staff and funds for developing informational and educational materials on venereal disease.

The project is based on several assumptions: Many individuals and groups who are apathetic about the increase in venereal disease, if drawn into a study of the local situation, would become concerned and, with professional guidance, could be stimulated to seek practical solutions. Also, it is believed that most young people in the Los Angeles area are ignorant as to the cause, spread, and cure of venereal disease and that most parents and school administrators would welcome help on an educational program.

The objectives of the Los Angeles program are to:

1. Promote junior and senior high school instruction on venereal disease by properly prepared teachers backed by informed administrators.

2. Determine current curriculum content and educational practices in the teaching of venereal disease.

3. Determine the level of knowledge and understanding pupils, teachers, and school administrators have about venereal disease.

4. Develop a comprehensive educational pro- gram on venereal disease for junior and senior high school teachers, administrators, school health and ancillary personnel, and parents.

5. Organize a communitywide supportive committee of agency representatives and interested persons who are or should be concerned about venereal disease.

The help of community leaders will be sought in the campaign against teenage venereal disease. The health departments will work with community agencies and organizations conducting youth and adult health and welfare programs and will offer agencies, groups, and individuals the opportunity to participate in a public health action program to provide the community with venereal disease information.

6. Promote community support for increased venereal disease instruction in the schools.

7. Plan seminars, workshops, postgraduate courses, and institutes for lay and professional groups. These programs will provide opportunities for school staff, medical, nursing, and other professional groups to participate actively in information and educational programs on venereal disease. Inservice training of all health department professional staff is envisaged.

8. Develop a general information program for the community concerning the effects of venereal disease on individual and community health. Increased use of mass media is planned. New and varied audiovisual aids and materials will be used.

9. Develop informational materials adapted to the local scene for instructional use in the schools and the community. Some informational materials in Spanish will aid in fulfillment of this objective.

10. Develop procedures for evaluating all phases of the venereal disease program.

-Mrs. Frank Shapiro, member of the Los Angeles City Board of Health Commissioners. Excerpted from a paper presented at a venereal disease seminar conducted by the Public Health Service, September 1963, at Santa Monica, Calif. 